\title{
Prevalência de sobrepeso, obesidade e alterações de pressão arterial em crianças do ensino fundamental de uma escola privada em Belém- PA
}

\author{
Prevalence of overweight, obesity and blood pressure changes in elementary school \\ children of a private school in Belém-PA
}

Prevalencia de sobrepeso, obesidad y cambios en la presión arterial en niños de primaria de una escuela privada en Belém-PA

Adilson Mendes de Figueiredo Júnior ${ }^{1 *}$, Lillian Nogueira da Costa ${ }^{1}$, Iracildo da Conceição dos Santos $^{1}$, Clebson Luis Trindade da Silva ${ }^{1}$, Bárbara Alves Ruela de Azevedo², Cipriana Catarina Aguiar $^{2}$, Fábio da Costa Ferreira², Malena Menezes Dantas Coelho ${ }^{3}$, Jussivan Oliveira do Nascimento ${ }^{3}$, Ana Carolina Vinagre Valle ${ }^{3}$, Carla Caroline Lobo de Souza ${ }^{4}$, Tereza Cristina dos Reis Ferreira $^{4}$, Maira Cibelle da Silva Peixoto ${ }^{4}$, Thiago Santos do Amaral ${ }^{4}$, Thayse Hage Gomes Machado ${ }^{5}$.

\section{RESUMO}

Objetivo: Verificar a prevalência do sobrepeso, obesidade, pressão arterial elevada e sedentarismo em crianças do $1^{\circ}$ ao $5^{\circ}$ ano do ensino fundamental de uma escola da rede privada. Métodos: Trata-se de um estudo observacional transversal. Participaram do estudo 139 estudantes, com faixa etária entre 6 a 11 anos, de ambos os sexos, que estavam devidamente matriculados em uma escola da rede privada na cidade de Belém (PA) através de amostra de conveniência. Resultados: 0 excesso de peso foi identificado em 40 $(28,8 \%)$ crianças, o sedentarismo foi identificado em $39(28,1 \%)$ crianças, a pressão arterial elevada foi identificada em 10 (7,2\%) alunos. Entre os 39 alunos sedentários encontramos $6(60 \%)$ hipertensos, e dos 129 não hipertensos 33 (26\%) são sedentários. Dos 39 alunos sedentários 12(46,2\%) estão com sobrepeso e $9(64,3 \%)$ são obesos. Conclusão: O sedentarismo pode estar correlacionado com o excesso de peso bem como a pressão arterial elevada, mostrando que crianças nestas faixas etárias, quando menos ativas fisicamente podem ter pressão arterial mais elevada e maior índice de massa corporal.

Palavras-chave: Obesidade, Epidemiologia, Crianças.

\begin{abstract}
Objective: To verify the prevalence of overweight, obesity, high blood pressure and physical inactivity in children from 1st to 5th grade of elementary school in a private school. Methods: This is a cross-sectional observational study. The study included 139 students, aged between 6 and 11 years, of both sexes, who were properly enrolled in a private school in the city of Belém (PA) through a convenience sample. Results: Overweight was identified in $40(28.8 \%)$ children, physical inactivity was identified in $39(28.1 \%)$ children, high blood pressure was identified in $10(7.2 \%)$ students. Among the 39 sedentary students we found $6(60 \%)$
\end{abstract}

${ }^{1}$ Escola Superior da Amazônia (ESAMAZ), Belém-Pará. *E-mail: adilsonmdfj@hotmail.com

2 Universidade Federal do Pará (UFPA), Belém-Pará.

${ }^{3}$ Centro Universitário do Pará (CESUPA), Belém-Pará.

${ }^{4}$ Universidade do Estado do Pará (UEPA), Belém-Pará.

${ }^{5}$ Universidade da Amazônia (UNAMA), Belém-Pará.

SUBMETIDO EM: 9/2019

ACEITO EM: 9/2019

PUBLICADO EM: 10/2019

REAS/EJCH | Vol.Sup.35 | e1691 | DOI: https://doi.org/10.25248/reas.e1691.2019 Página 1 de 8 
hypertensive, and of the 129 non-hypertensive $33(26 \%)$ are sedentary. Of the 39 sedentary students 12 $(46.2 \%)$ are overweight and $9(64.3 \%)$ are obese. Conclusion: Physical inactivity may be correlated with overweight as well as high blood pressure, showing that children in these age groups, when less physically active, may have higher blood pressure and higher body mass index.

Keywords: Obesity, Epidemiology, Children.

\section{RESUMEN}

Objetivo: Verificar la prevalencia de sobrepeso, obesidad, presión arterial alta e inactividad física en niños de $1^{\circ}$ a $5^{\circ}$ grado de primaria en una escuela privada. Métodos: Este es un estudio observacional transversal. El estudio incluyó a 139 estudiantes, de entre 6 y 11 años, de ambos sexos, que se matricularon adecuadamente en una escuela privada en la ciudad de Belém (PA) a través de una muestra de conveniencia.

Resultados: Se identificó sobrepeso en 40 (28.8\%) niños, se identificó inactividad física en 39 (28.1\%) niños, se identificó presión arterial alta en $10(7.2 \%)$ estudiantes. Entre los 39 estudiantes sedentarios encontramos $6(60 \%)$ hipertensos, y de los 129 no hipertensos $33(26 \%)$ son sedentarios. De los 39 estudiantes sedentarios, $12(46,2 \%)$ tienen sobrepeso y $9(64,3 \%)$ son obesos. Conclusión: La inactividad física puede estar correlacionada con el sobrepeso y la presión arterial alta, lo que demuestra que los niños en estos grupos de edad, cuando son menos activos físicamente, pueden tener una presión arterial más alta y un índice de masa corporal más alto.

Palabras-clave: Obesidad, Epidemiología, Niños.

\section{INTRODUÇÃO}

Nas últimas décadas observa-se uma ascensão das doenças crônico-degenerativas, especialmente as cardiovasculares em nível global. Está realidade pode estar sendo explicada pela manifestação do processo de globalização no estilo de vida das populações de diversos países, a qual acarreta a mudança da dieta e da composição corporal dos indivíduos. Dados do ano de 2009 ofertados pelo Ministério da Saúde relatam que as doenças cardiovasculares são as principais causas de morte no Brasil e que a prevenção e controle dos fatores de risco ainda são os meios mais efetivos de redução das taxas de mortalidade. Sobrepeso e obesidade são fatores que contribuem no desenvolvimento das doenças cardiovasculares e quanto mais cedo forem adquiridos pelo indivíduo maior será sua repercussão no estado geral de saúde do mesmo (GUEDES PD, et al. 2006; MANSUR AP e FAVARATO D, 2012; ARAÚJO MFM, et al. 2010).

As doenças crônicas não transmissíveis (DCNT) ainda correspondem a $63 \%$ de todas as causas de óbito no mundo e entre elas destacam-se as doenças cardiovasculares pela sua magnitude que representa um terço das mortes globais. Estima-se que cerca de 600 milhões de pessoas tenham hipertensão arterial e que ocorram 7,1 milhões de mortes ocorram no planeta. Principalmente, porque conduzem ao maior risco para desenvolvimento de doenças fatais e não fatais, insuficiência renal e outras (MALTA DC et al, 2017).

Há uma relação direta entre a pressão arterial e o excesso de peso, na qual pessoas obesas tendem a se tornar hipertensas, devido o fato da obesidade ser considerada um estado pré-hipertensivo e gerar hiperatividade simpática, reter sódio, expandir o volume intravascular e diminuir o débito cardíaco. A prevenção ainda é a melhor forma de combater a hipertensão e por este motivo preconizou-se que fosse realizada a verificação anual da pressão arterial em crianças acima de três anos de idade, segundo a VI Diretrizes Brasileiras de Hipertensão (CUREAU FV e REICHERT FF, 2013; FERNANDO P, 1994).

Sobrepeso e obesidade estão relacionados há uma série de causas como inatividade física, hábitos alimentares pouco saudáveis, predisposição genética e sua prevalência vária conforme a idade, cor, sexo, raça e classe socioeconômica. A prevalência do excesso de peso e da obesidade vem crescendo na infância; fase na qual são formados os hábitos alimentares e de atividade física e cujas pesquisas demonstram ser a fase de início de processos patológicos como arteriosclerose e hipertensão arterial. 
Atualmente, a obesidade apresenta maiores taxas de prevalência no mundo e no Brasil. Nos Estados Unidos, cerca 20 e $27 \%$ das crianças e adolescentes são afetadas pela obesidade e no Brasil a obesidade em menores de cinco anos varia de 2,5\% entre as crianças de baixa renda e de $10,6 \%$ em crianças de renda maior (SILVA GAP, BALABAN G e MOTTA MEF, 2005).

Sendo assim a importância da prevenção, diagnóstico e tratamento do sobrepeso e obesidade voltado para infância (ABRANTES MM, et al. 2002; MORAES ACF, et al. 2007).

Diante disso, o objetivo geral deste estudo foi verificar a prevalência do sobrepeso, obesidade, pressão arterial elevada e sedentarismo em crianças do $1^{\circ}$ ao $5^{\circ}$ ano do ensino fundamental de uma escola da rede privada.

\section{MÉTODOS}

Trata-se de um estudo observacional transversal. Participaram do estudo 139 estudantes através de amostra de conveniência, com faixa etária entre 6 a 11 anos, de ambos os sexos, que estavam devidamente matriculados em uma escola da rede privada na cidade de Belém (PA). Foram excluídos os estudantes cujo responsável não assinou o Termo de Consentimento Livre e Esclarecido, os estudantes que não assentiram com a pesquisa e os que apresentaram doença cardíaca. A coleta de dados foi realizada de forma individual com a presença de um responsável pelo colégio entre os meses de outubro e novembro no ano de 2014. Neste estudo foram avaliadas as seguintes variáveis: pressão arterial sistêmica, "risco de sobrepeso/ obesidade" e o sedentarismo.

Para avaliação da pressão arterial sistêmica utilizou-se um esfigmomanômetro com braçadeira para avaliação infantil e estetoscópio pediátrico, ambos da marca Tycos ( $\mathrm{KOCH} \mathrm{VH,} \mathrm{2000),} \mathrm{sendo} \mathrm{considerada}$ pressão arterial sistêmica elevada quando apresentava valores iguais ou superiores ao percentil 95, tendo como base o estudo National High Blood Pressure Education Program em 2004.

Para obtenção do "risco de sobrepeso e obesidade" foi necessário realizar o cálculo do Índice de Massa Corporal, pois através do valor do mesmo é possível classificar o sobrepeso e obesidade tendo como base a escala de Cole TJ et al. (2000). A obtenção das medidas antropométricas (peso e altura) seguiu a padronização prevista na organização mundial de saúde.

Buscou-se analisar a realização de atividades físicas pelos estudantes utilizando o questionário Godin Shephard Leisure - Time Physical Activity Questionnaire (GSLTPAQ), a fim de definir o tempo destinado á prática de atividades físicas e definir a presença ou ausência de sedentarismo nos escolares.

A análise estatística das variáveis foi realizada utilizando o software BioEstat 5.0. Inicialmente, realizou-se a análise descritiva dos dados por meio do teste de Kolmogorov Smirnov. Os resultados foram apresentados por meio de tabelas e valores absolutos e relativos. Foi utilizado o test $\mathrm{t}$ de Student com um nível de significância de $95 \%$.

A casuística inicial era de 152 alunos regularmente matriculados. De acordo com os critérios de inclusão e exclusão; doença cardíaca, assinatura do responsável legal e o assentimento da criança obtiveram-se como casuística final, 139 alunos. Esse número de alunos corresponde á 5 turmas de $1^{\circ}$ ao $5^{\circ}$ ano do ensino fundamental da escola estudada.

O estudo foi aprovado pelo Comitê de Ética e Pesquisa em Seres Humanos do Campus II da Universidade do Estado do Pará - CEP/UEPA (n 819.084/ 2014).

\section{RESULTADOS}

Das crianças avaliadas 76 foram do sexo feminino (54,7\%) e 63 do sexo masculino (45,3\%). Quanto à idade $42(30,2 \%)$ tinham entre 6 e 8 anos, $48(34,5 \%)$ entre 8 e 10 anos, $49(35,3 \%)$ tinham entre 10 e 11 anos. 
A análise das variáveis: Peso, altura, pressão arterial sistêmica (sistólica e diastólica) e do Godin Shephard Leisure Time Physical Activity Questionnaire (GSLTPAQ) pode ser feita a partir dos valores apresentados na Tabela 1, da qual pode se concluir entre outros fatores que a variáveis apresentam média de 32,25 - 1,32101,06- 68,73 - 37,44 respectivamente (Tabela 1).

Tabela 1 - Estatísticas descritivas das variáveis: Peso, Altura, Sistólica, Diastólica e GSLTPAQ das crianças entre 6 e 11 anos, realizada no ano de 2014.

\begin{tabular}{lllllll} 
Estatísticas & Média & Mediana & Desvio Padrão & Coeficiente de Variação & Mínimo & Máximo \\
& & & & & & \\
\hline Peso & 32,25 & 29 & 11,38 & $35,3 \%$ & 15,6 & 76,3 \\
Altura & 1,32 & 1,31 & 0,11 & $8,5 \%$ & 1,09 & 1,56 \\
Sistólica & 101,06 & 100 & 14,60 & $14,4 \%$ & 35 & 154 \\
Diastólica & 68,73 & 68 & 11,44 & $16,6 \%$ & 16 & 81 \\
GSLTPAQ & 37,44 & 37 & 11,14 & $29,8 \%$ & & \\
\hline
\end{tabular}

Fonte: Figueiredo Júnior AM, et al., 2014.

O excesso de peso foi identificado em 40 crianças, entre estas $26(18,7 \%)$ foram consideradas com sobrepeso sendo 14 (18\%) meninas e $12(19 \%)$ meninos. 14 (10,1\%) alunos estão com obesidade entre estes $7(9 \%)$ meninas e $7(11 \%)$ meninos. . O sedentarismo foi identificado em 39 (28.1\%) crianças, sendo $21(27,6 \%)$ do sexo feminino e $18(28,6 \%)$ do sexo masculino. A pressão arterial elevada foi identificada em 10 alunos $(7,2 \%), 8(12,7 \%)$ do sexo masculino e $2(2,6 \%)$ do sexo feminino, conforme indica a Tabela 2.

Tabela 2 - Cruzamento entre Sexo e Hipertensão, referente à pesquisa sobre Obesidade, sobrepeso, pressão arterial elevada e sedentarismo, realizada no ano de 2014.

\begin{tabular}{|c|c|c|c|c|c|c|}
\hline \multirow{2}{*}{ Sexo } & \multicolumn{4}{|c|}{ Hipertensão } & \multirow{2}{*}{ Total } & \multirow{2}{*}{$\%$} \\
\hline & Sim & $\%$ & Não & $\%$ & & \\
\hline Feminino & 2 & $2,6 \%$ & 74 & $97,4 \%$ & 76 & $54,7 \%$ \\
\hline Masculino & 8 & $12,7 \%$ & 55 & $87,3 \%$ & 63 & $45,3 \%$ \\
\hline Total & 10 & $7,2 \%$ & 129 & $92,8 \%$ & 139 & $100,0 \%$ \\
\hline
\end{tabular}

Fonte: Figueiredo Júnior AM, et al., 2014. Teste Exato de Fisher = 0,043 (Significativo).

A distribuição do sedentarismo quanto á idade foi: Entre 6 e 8 anos de $13(31 \%), 8-10$ anos de 10 (28,8\%), 10- 12 anos de 16 (32,7\%). A distribuição da pressão arterial elevada quanto á idade foi: Entre 6 e 8 anos de 2 (4,8\%), 8-10 anos de 2 (4,2\%), 10-12 anos de 6 (12,2\%). Quando cruzados percebe-se que os alunos sedentários são também hipertensos e apresentam excesso de peso. Entre os 39 alunos sedentários encontramos 6 (60\%) alunos hipertensos, e dos 129 não hipertensos 33(26\%) são sedentários (Tabela 3).

Tabela 3 - Cruzamento entre Hipertensão e Classificação, referente à pesquisa sobre Sobrepeso, obesidade, pressão arterial elevada e sedentarismo, realizada no ano de 2014.

\begin{tabular}{|c|c|c|c|c|c|c|}
\hline \multirow[b]{2}{*}{ Hipertensão } & \multicolumn{4}{|c|}{ Classificação } & \multirow[b]{2}{*}{ Total } & \multirow[b]{2}{*}{$\%$} \\
\hline & Pouco ativo ou Inativo & $\%$ & Ativo Regularmente & $\%$ & & \\
\hline$\overline{S i m}$ & 6 & $60 \%$ & 4 & $40 \%$ & 10 & $7,2 \%$ \\
\hline Não & 33 & $26 \%$ & 96 & $74 \%$ & 129 & $92,8 \%$ \\
\hline Total & 39 & $28 \%$ & 100 & $72 \%$ & 139 & $100,0 \%$ \\
\hline
\end{tabular}

Fonte: Figueiredo Júnior AM, et al., 2014.Teste Exato de Fisher = 0,0293 (Significativo) 
Dos 39 alunos sedentários $12(46,2 \%)$ estão com sobrepeso e $9(64,3 \%)$ são obesos. Dos 100 não sedentários 19 estão com excesso de peso sendo 14(53,8\%) com sobrepeso e 5 (35,7\%) obesos (Tabela 4).

Tabela 4 - Cruzamento entre IMC e Classificação, referente à pesquisa sobre Sobrepeso, obesidade, pressão arterial elevada e sedentarismo realizada no ano de 2014.

\begin{tabular}{llllllll}
\hline & \multicolumn{5}{c}{ Classificação } & & \\
\cline { 2 - 6 } IMC & Pouco ativo ou Inativo & $\%$ & Ativo Regularmente & $\%$ & Total & $\%$ \\
& & & & & & & \\
\hline Normal & 18 & $18,2 \%$ & 81 & $81,8 \%$ & 99 & $71,2 \%$ \\
Sobrepeso & 12 & $46,2 \%$ & 14 & $53,8 \%$ & 26 & $18,7 \%$ \\
Obeso & 9 & $64,3 \%$ & 5 & $35,7 \%$ & 14 & $10,1 \%$ \\
\hline Total & 39 & $28,1 \%$ & 100 & $71,9 \%$ & 139 & $100,0 \%$ \\
\hline
\end{tabular}

Fonte: Figueiredo Júnior AM, et al., 2014. $G=16,3652 p=0,003$ (Altamente Significativo).

Não foi encontrada relação estatisticamente significaticante através do teste estatístico Qui - Quadrado entre o excesso de peso e pressão arterial elevada. Dos 99 com IMC normal 4 estão com pressão elevada. De 26 alunos com sobrepeso 3 (11,5\%) estão com pressão arterial elevada, dos 14 com obesidade $3(21,4 \%)$ estão com pressão elevada (Tabela 5).

Tabela 5 - Cruzamento entre IMC e Hipertensão, referente à pesquisa sobre Sobrepeso, obesidade, pressão elevada e sedentarismo, realizada no ano de 2014.

\begin{tabular}{|c|c|c|c|c|c|c|}
\hline \multirow{2}{*}{ IMC } & \multicolumn{4}{|c|}{ Hipertensão } & \multirow{2}{*}{ Total } & \multirow{2}{*}{$\%$} \\
\hline & Sim & $\%$ & Não & $\%$ & & \\
\hline Normal & 4 & $4,0 \%$ & 95 & $96,0 \%$ & 99 & $71,2 \%$ \\
\hline Sobrepeso & 3 & $11,5 \%$ & 23 & $88,5 \%$ & 26 & $18,7 \%$ \\
\hline Obeso & 3 & $21,4 \%$ & 11 & $78,6 \%$ & 14 & $10,1 \%$ \\
\hline Total & 10 & $7,2 \%$ & 129 & $92,8 \%$ & 139 & $100,0 \%$ \\
\hline
\end{tabular}

Fonte: Figueiredo Júnior AM, et al., 2014. $G=4,6393 p=0,0983$ (Não Significativo).

\section{DISCUSSÃO}

Sobrepeso e obesidade estão relacionados há uma série de causas e tornaram-se problemas de saúde publica em países desenvolvidos e em desenvolvimento. Pessoas obesas geralmente apresentam baixa autoestima e dificuldade em relaciona-se. Outros fatores importantes são: a sua associação com outras doenças como: doença cardíaca, osteoartrite, diabetes tipo 2 e alguns tipos de câncer (ABRANTES MM, et al. 2002).

Dados epidemiológicos demonstram que houve um aumento de $54 \%$ na prevalência da obesidade entre crianças e adolescentes nos últimos 20 anos (MORAES ACF, et al. 2007).

A atividade física é fator importante na composição corporal dos indivíduos, já que aumenta a massa óssea e preveni a osteoporose e a obesidade (MELLO DE, et al. 2004).

Quando os níveis de realização da atividade física decrescem seja no tempo livre ou em lazer, temos o sedentarismo implantado. $\mathrm{Na}$ atualidade a atividade física com grande consumo energético como pular e correr vem sendo cada vez menos praticada e substituída por atividades com menor gasto energético 
(navegar na internet, jogar vídeo game assistir televisão), principalmente nas fases da infância e adolescência (MACHADO YL, 2011).

O sedentarismo é precursor da obesidade em crianças, além disso, a obesidade por si só tende há estimular o sedentarismo ( $\mathrm{KOCH} \mathrm{VH,} \mathrm{2000).} \mathrm{Atividades} \mathrm{físicas} \mathrm{ativas} \mathrm{(deslocar-se} \mathrm{para} \mathrm{a} \mathrm{escola} \mathrm{e} \mathrm{brincar}$ e/ou praticar exercícios formais) tem menor aceitação quando comparadas á atividades sedentárias (televisão e/ou videogame) entre muitos escolares e que quanto mais ativa for a criança menor será o seu percentual de gordura e seu IMC (BARUKI SBS, et al. 2006).

O IMC tende a ser transformado de forma constante mediante a mudança na idade A precocidade de instalação da obesidade na faixa etária entre 4 a 8 anos faz com que haja permanência da incidência da mesma na vida adulta, contudo existem três períodos em que se deve evitar a incidência desta patologia, são eles: primeiro ano de vida; entre os 5 a 7 anos de idade e entre 6 a 11 anos (OLIVEIRA AMA, et al. 2003).

As diversas evidências da literatura relatam maior prevalência da obesidade nas mulheres tanto na infância quanto na vida adulta (MORAES ACF, et al. 2007). Os resultados encontrados não demonstram diferenças estatisticamente significantes no que se diz respeito ao IMC quando analisamos o sexo $(p=0,9247)$ e a faixa etária, ou seja, nesta variável houve distribuição equilibrada do excesso de peso em meninas e meninos de diferentes grupos etários. Analisou-se o excesso de peso á nível global podemos observar uma crescente ascensão da obesidade. Entre os anos de 1963 a 1980, nos EUA, na faixa de 6 a 11 anos, a obesidade cresceu aproximadamente 18 a $30 \%$ nos meninos e 17 a $25 \%$ nas meninas. No Japão, a obesidade, em crianças de 10 anos, cresceu de $3-3,5 \%$, em 1968, para 8 -9\%, em 1992 (PIMENTA APAA e PALMA A, 2001).

No Brasil, segundo Radominski RB (2011) houve maior prevalência de índices de sobrepeso em áreas urbanas e em classes com maior renda per capita, comprovando que entre os anos de 2008 e 2009: 16,6\% dos meninos e $11,8 \%$ das meninas brasileiras na faixa etária de 5 a 9 anos foram considerados obesos. Outro ponto importante foi a constatação da má nutrição mesmo nas classes com menor renda, onde ocorreu uma triplicação do excesso de peso (de 8, 9\% para 26,5\%).

Neste estudo, a mostra apresentou taxas de sedentarismo e excesso de peso isso demonstra que a amostra obtida é significante $(p=0,003)$ o número de crianças com excesso de peso analisado pelo IMC e que fazem o mínimo possível de movimento implicando em risco a saúde das mesmas. Em relação à pressão arterial elevada é mais prevalente em homens do que me mulheres e em pessoas brancas (MICHEL L, 2002); e tem sido determinada por alguns fatores, entre os quais a obesidade, o uso de sódio na dieta, as angústias, tensão emocional e a hereditariedade, já que filhos de pais hipertensos têm mais chance de desenvolver hipertensão.

Ou seja, a presença de hipertensão na população se dá por uma interação entre predisposição poligênica e fatores ambientais diversos (FUJIMURA MD, 1983). A pressão arterial em crianças e adolescentes tem estado cada vez mais em foco. Os estudos recentes têm mostrado uma variação de $1 \%$ a $13 \%$, dependendo dos critérios metodológicos, contudo as maiores prevalências são encontradas nos estudos que tem por base uma única visita única (FUJIMURA MD, 1983).

A dieta com alto teor de sal, inatividade física e excesso de peso vem sendo responsabilizados por está tendência nas últimas décadas (CAMPANA GME, et al. 2009). No Brasil, estudo realizado por Fujimura MD (1983) com 7.015 crianças e adolescentes realizado na cidade do Rio de Janeiro, com faixa etária entre 6 e 15 anos a qual teve como resultado uma relação direta entre a pressão arterial e o peso corporal (pré hipertensão em crianças e adolescentes). Até o momento não foi elucidado um número satisfatórios de estudos no Brasil que abordem temas relacionados à pressão arterial nas duas primeiras décadas de vida $\mathrm{e}$ os que existem diferem na metodologia empregada, o que mostra uma enorme carência de investigações neste segmento da população (MAGALHÃES MEC, et al. 2002).

A pressão alta na amostra estudada apresentou maior prevalência no sexo masculino quando comparado ao feminino, sendo que não se observou variações da pressão arterial nos diferentes grupos etários $(p=$ 
0,2748). Dos 39 alunos sedentários 6 apresentaram pressão alta, apresentando diferença estatisticamente significante $(p=0,0293)$, correlacionando com o sedentarismo (para o mesmo grupo), pode ser sugestivo de fator agravante no estado de saúde geral dos indivíduos.

\section{CONCLUSÃO}

Conclui-se que para a população pesquisada há baixa prevalência de excesso de peso (sobrepeso, obesidade) e sedentarismo sem diferenças estatisticamente significante entre os sexos e faixa etária. Quanto à pressão alta obteve-se diferença significante entre o sexo (predomínio no masculino 12,7\%). Como limitações citamos que a hipertensão não se pode avaliar, pois só foi realizada uma aferição diária, apresentando 10 alunos com a pressão elevada no momento da avaliação, mas como benefícios ressalta-se que tais dados podem servir para a efetivação de futuros protocolos e políticas públicas em saúde.

\section{REFERÊNCIAS}

1. ABRANTES MM, et al. Prevalência de sobrepeso e obesidade em crianças e adolescentes das regiões sudeste e nordeste. Jornal de pediatria. 2002; (78)4: 335-40.

2. ARAUJO MFM, et al. Sobrepeso entre adolescentes de escolas particulares de Fortaleza, CE, Brasil. Revista Brasileira de enfermagem. 2010; (63)4: 623-28.

3. BARUKI SBS, et al. Associação entre estado nutricional e atividade física em escolares da rede municipal de ensino em Corumbá - MS. Rev. Bras. Med. Esporte. 2006; 12(2): 90-4.

4. CAMPANA GME, et al. Pré hipertensão em crianças e adolescentes. Rev Bras Hipertens. 2009; 16(2): 92-102.

5. COLE TJ, et al. Establishing a standard definition for child overweight and obesity worldwide: international survey. BMJ 2000; 320:1240-3.

6. CUREAU FV, REICHERT FF. Indicadores antropométricos de obesidade como preditores de pressão arterial elevada em adolescentes. Rev Bras Cineantropom Desempenho Hum. 2013; (15)3: 338-349.

7. FERNANDO P. Risco cardiovascular: Fatores metabólicos e nutricionais: Diagnóstico e tratamento. São Paulo: $A S T A$ Médica; 1994. p. 103-121.

8. FUJIMURA MD. Hipertensão arterial na infância: Diagnóstico e tratamento. Pediatria. São Paulo: Livros médicos Ltda; 1983; (5): 205-13.

9. GUEDES PD, et al. Prevalência de sobrepeso e obesidade em crianças e adolescentes: estimativas relacionadas ao sexo, à idade e à classe socioeconômica. Rev. bras. Educ. Fís. Esp. 2006; (20)3: 151-63.

10. HESS ML. Doenças cardíacas: Primeiros cuidados. São Paulo: Manole; 2002. p. 80-107.

11. $\mathrm{KOCH}$ VH. Pressão arterial em Pediatria: Aspectos metodológicos e normatização. Rev Bras de Hipertens. 2000; (7) 1: 71-8.

12. MACHADO YL. Sedentarismo e suas consequências em crianças e adolescentes [dissertação]. Minas Gerais: Instituto Federal de Educação Ciências e Tecnologia do Sul de Minas, Campus Muzambinho; 2011.

13. MAGALHÃES MEC, BRANDÃO AA, POZZAN R, BRANDÃO AP. Hipertensão arterial em crianças e adolescentes. Rev Bras Hipertens. 2002; (9)3: 245-255.

14. MALTA DC, et al. Prevalência e fatores associados com hipertensão arterial autorreferida em adultos rasileiros. Rev. Saúde Pública. 2017.

15. MANSUR AP, FAVARATO D. Mortalidade por doenças cardiovasculares no Brasil e na região metropolitana de São Paulo: Uma atualização. Arq Bras Cardiol. 2012; (99)2: 755-761.

16. MELLO DE, et al. Obesidade infantil; como podemos ser eficazes. Jornal de Pediatria. 2004; (80)3: $173-82$.

17. MORAES ACF, et al. Prevalência de sobrepeso e obesidade em alunos do ensino fundamental da cidade de Maringá-PR. Cienc Cuid Saude. 2007; (6)2: 364-369.

18. National High Blood Pressure Education Program Working Group on High Blood Pressure in children adn Adolescents. The fourth report on the diagnosis, evaluation, and tretment of high blood pressure $\mathrm{i}$ children and adolescents. Pediatrics. 2004; 1(14): 555-79.

19. OLIVEIRA AMA, et al. Sobrepeso e obesidade infantil: influência de fatores biológicos e ambientais em Feira de Santana, BA. Arq Bras Endocrinol Metab. 2003; (47)2: 144-50.

20. PIMENTA APAA, PALMA A. Perfil epidemiológico da obesidade em crianças: relação entre televisão, atividade física e obesidade. Rev. Bras. Ciĺn. e Mov. Brasília. 2001; 9(4): 19-24.

21. RADOMINSKI RB. Aspectos epidemiológicos da obesidade infantil. 49 ed. Paraná, Revista da Associação Brasileira para o Estudo da Obesidade e da Síndrome metabólica. 2011; 49. 
22. SILVA GAP, BALABAN G e MOTTA MEF. Prevalência de sobrepeso e obesidade em crianças e adolescentes de diferentes condições socioeconômicas. Rev. Bras. Saúde Matern. Infant., Recife. 2005; 5(1): 53-59.

23. WHO (World Health Organization). Physical Status: the use and interpretation of anthropometry - Report of a WHO Expert Committee. Geneva 1995. 